\title{
Twinning in magnesium under dynamic loading
}

\author{
Neha Dixit ${ }^{1}$, Kavan Hazeli ${ }^{2}$, and Kaliat T. Ramesh ${ }^{1,2, a}$ \\ ${ }^{1}$ Department of Mechanical Engineering, Johns Hopkins University, Baltimore, MD, USA \\ ${ }^{2}$ Hopkins Extreme Materials Institute, Johns Hopkins University, Baltimore, MD, USA
}

\begin{abstract}
Twinning is an important mode of deformation in magnesium $(\mathrm{Mg})$ and its alloys at high strain rates. Twinning in this material leads to important effects such as mechanical anisotropy, texture evolution, tension-compression asymmetry, and sometimes non-Schmid effects. Extension twins in Mg can accommodate significant plastic deformation as they grow, and thus twinning affects the overall rate of plastic deformation. We use an experimental approach to study the deformation twinning mechanism under dynamic loading. We perform normal plate impact recovery experiments (with microsecond pulse durations) on pure polycrystalline Mg specimens. Estimates of average TB velocity under the known impact stress are obtained by characterization of twin sizes and aspect ratios developed within the target during the loading pulse. The measured average $\mathrm{TB}$ velocities in our experiments are of the order of several $\mathrm{m} \mathrm{s}^{-1}$. These velocities are several orders of magnitude higher than those so far measured in $\mathrm{Mg}$ under quasi-static loading conditions. Electron back-scattered diffraction (EBSD) is then used to characterize the nature of the twins and the microstructural evolution. Detailed crystallographic analysis of the twins enables us to understand twin nucleation and growth of twin variants under dynamic loading.
\end{abstract}

\section{Introduction}

The low density, high specific strength (strength/weight ratio) [1], and good damping properties [2,3] of $\mathrm{Mg}$ and its alloys makes these materials attractive for applications such as automobile and aerospace components. For successful use of these materials in applications in which dynamic loading conditions (e.g. crash) are expected, an understanding of the deformation mechanisms under dynamic loading is important. Dynamic loading generally involves varying strain rates, short time durations and complex stress states. It is known that the stress-strain response of $\mathrm{Mg}$ alloys is highly anisotropic as well as sensitive to strain rate [4]. Tucker et al. [5] reported strong strain rate dependences of the compressive yield, strain hardening rate and ductility for loading along the normal direction of a rolled $\mathrm{Mg}$ sheet. In the rolling and transverse directions, no strain rate dependence of the yield strength was observed. Instead a marked increase in strain hardening was observed beginning at a characteristic strain level. Agnew et al. [6] investigated the effect of rare-earthelements on the ballistic properties of Mg alloys using a Kolsky bar technique. This study demonstrated that the effect of strain rate on the yield strength of a textured $\mathrm{Mg}$ WE43B-T5 alloy is small and the strain-hardening behavior is only mildly rate sensitive. The review article by Prasad et al. [7] suggested that the flow stress at yield is relatively insensitive to strain rate in the cases where deformation twinning was the dominant mechanism, but the flow stress exhibits some sensitivity to the strain rate when slip was the dominant deformation mechanism. Despite the great interest and importance of deformation mechanisms associated with dynamic behavior, there are

\footnotetext{
${ }^{a}$ Corresponding author: ramesh@jhu.edu
}

limited studies on the dynamic response of pure $\mathrm{Mg}$ and very little information on the relative contributions to plastic deformation of twinning versus slip as a function of strain rate. Our objective is to build a fundamental understanding of the dynamic deformation of $\mathrm{Mg}$ and its alloys. We begin by focusing on pure $\mathrm{Mg}$ as a model material in this study. The insights gained from this work can then perhaps be applied to the analysis of the dynamic behavior of various $\mathrm{Mg}$ alloys.

\section{Normal plate impact experiments}

A hot-extruded $\mathrm{Mg}$ rod of commercial purity $(99.9 \%)$ was used in this study. The microstructure of the material consists of equiaxed grains with an average grain size of about $20 \mu \mathrm{m}$. Normal plate impact experiments were performed, with impact in the extrusion direction at impact velocities of $60-70 \mathrm{~m} / \mathrm{s}$. At this velocity, there is elasticplastic wave propagation in the material but no shocks are generated. In the normal plate impact experiment, a cylindrical plate of material (called the flyer) is carried on a projectile that is launched at high velocities, producing an impact onto a stationary plate (called the target) that is supported in a target holder within an evacuated target chamber. The two plates are parallel to each other and perpendicular to the direction of projectile motion, and thus the impact causes longitudinal wave propagation in both plates.

A schematic of the wave propagation in the plates is shown in the Lagrangian diagram of Fig. 1. Upon impact, longitudinal elastic and plastic waves are generated in both flyer and target plates. This happens when the impact velocity generates stresses above the yield strength of the material in this uniaxial strain configuration. The

This is an Open Access article distributed under the terms of the Creative Commons Attribution License 4.0, which permits unrestricted use, distribution, and reproduction in any medium, provided the original work is properly cited. 


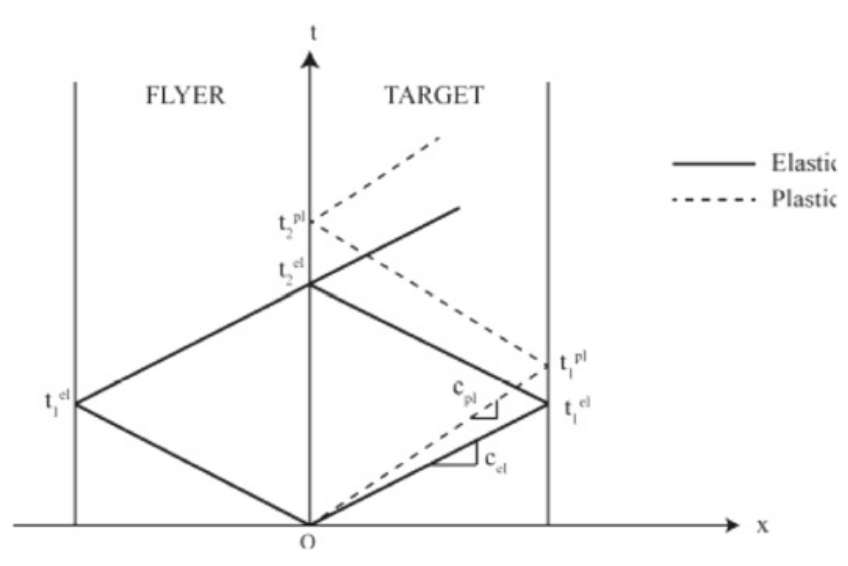

Figure 1. Elastic-plastic wave propagation in the symmetric normal plate impact configuration (the plastic wave is not shown in the flyer for simplicity).

elastic wave represented by a solid line travels at a larger speed $\left(c_{\mathrm{el}}\right)$ than the plastic wave $\left(\mathrm{c}_{\mathrm{pl}}\right)$ represented by the dashed line. Both these waves travel through the thickness of the plate to the rear free surface. The rear surface has a stress-free boundary condition, and therefore the reflected waves from the free surface are of opposite sign. Similar wave propagation occurs in the flyer plate as well, but only the elastic wave in the flyer is shown in the figure for simplicity. The reader interested in the plate impact technique is referred to the following article in the Handbook of Experimental Solid Mechanics [8].

The flyer and the target plates in these experiments are both made of pure $\mathrm{Mg}$ and are of the same dimensions (25.4 mm diameter, $3.5 \mathrm{~mm}$ thickness) for this symmetric impact. Therefore, the reflected waves from the rear surfaces of the flyer and target plates will arrive at the impact face at approximately the same time and therefore unload each other. Note that the normal plate impact experiment imposes a condition of uniaxial strain at the centre of the plate before unloading (release) waves from the edges arrive at the point being interrogated on the rear surface of the target. The experimental measurements consist of the projectile velocity before impact (measured using a Laser Line Velocity Sensor), the tilt/misalignment between the impacting plates (measured using tilt pins) and the normal particle displacement at the free surface of the target (measured using a laser-based Normal Displacement Interferometer).

The density of $\mathrm{Mg}$ is $1740 \mathrm{~kg} / \mathrm{m}^{3}$, the elastic Young's modulus is $44 \mathrm{GPa}$ and the Poisson's ratio is 0.35 [9]. The elastic longitudinal wave speed in $\mathrm{Mg}$ is $\mathrm{c}_{\mathrm{el}}=6370 \mathrm{~m} / \mathrm{s}$, and so the round trip travel time for the $3.5 \mathrm{~mm}$ thick plate is $1.1 \mu \mathrm{s}$ for the elastic wave $\left(\mathrm{t}_{2}^{\mathrm{el}}\right)$. The effective pulse duration for this experiment is the time needed for the plastic wave to arrive again at the impact face after traveling through the target thickness $\left(\mathrm{t}_{2}^{\mathrm{pl}}\right)$, and is about $1.3 \mu \mathrm{s}$. The plates are recovered after impact and examined using optical and electron microscopy to determine the deformation mechanisms active within these loading times.

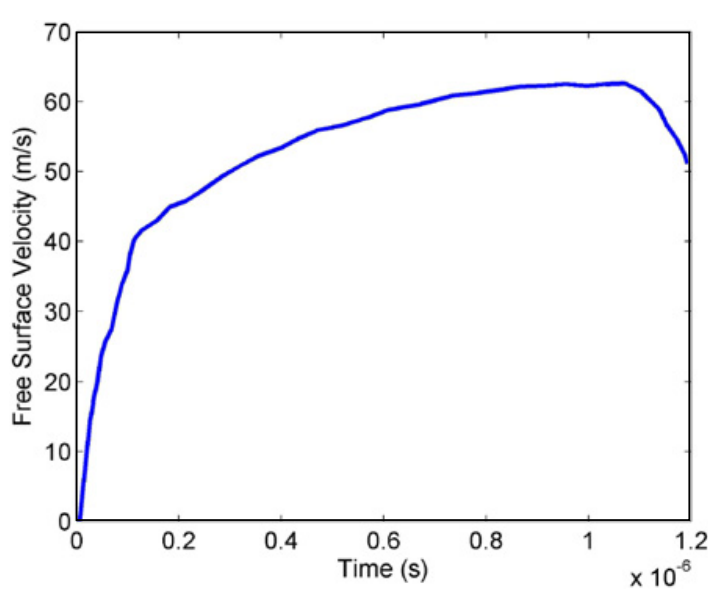

Figure 2. Experimentally measured particle velocity history at the free surface of a $3.5 \mathrm{~mm}$ target after an initial impact at $70 \mathrm{~m} \mathrm{~s}^{-1}$.

\section{Results and discussion}

\subsection{Free surface velocity}

The particle displacement at the free surface of the target is measured by the normal displacement interferometer (NDI), and is then numerically differentiated to obtain the rear surface particle velocity. An example of the free surface velocity history for a target plate of $3.5 \mathrm{~mm}$ thickness is shown in Fig. 2. There is a rapid increase in the free surface velocity (Fig. 2) at first in response to the arrival of the elastic wave, and then the rate of increase of the velocity slows down as plasticity sets in. Due to the wave propagation associated with the plate impact experiment, the stress, strain and strain rates are inhomogeneous throughout the thickness of the plates and evolve with time at each location. Computational estimates of the dynamic plastic deformations [REF Neha's thesis] suggest that the local plastic strain rates are of the order of $10^{4}-10^{5} \mathrm{~s}^{-1}$ in these experiments, but these high strain rates are present in specific regions of the plates for only small fractions of a microsecond.

We now examine the deformation mechanisms active within the target plate during this dynamic loading.

\subsection{Microstructural evolution}

The deformed target plates were recovered after the plate impact experiment and sectioned by electrical discharge machining (EDM) to make samples for microstructural analysis. The microstructure of these targets was analysed using electron back-scattered diffraction (EBSD), with the focus on the characterization of the observed twins (dislocations cannot be effectively observed using this method, and TEM results on the dislocation motion will be reported in subsequent work.

Note that $\mathrm{Mg}$ has two commonly observed modes of twinning, leading to extension and contraction twins [10, 11]. In addition to primary twinning, secondary twinning can occur within the reoriented primary twins, known as double twinning [12]. The material being tested has an initial texture as a consequence of the extrusion. As a consequence, compressive loading along this direction 


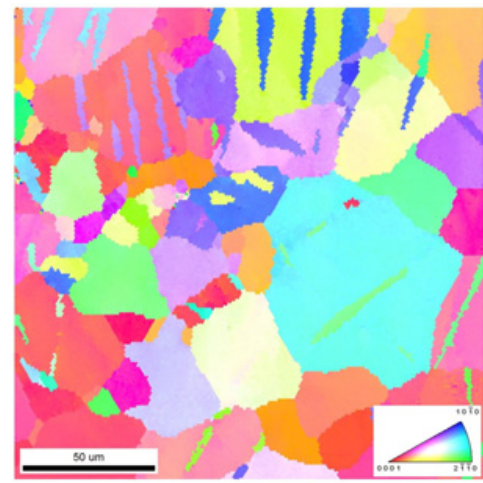

(a)

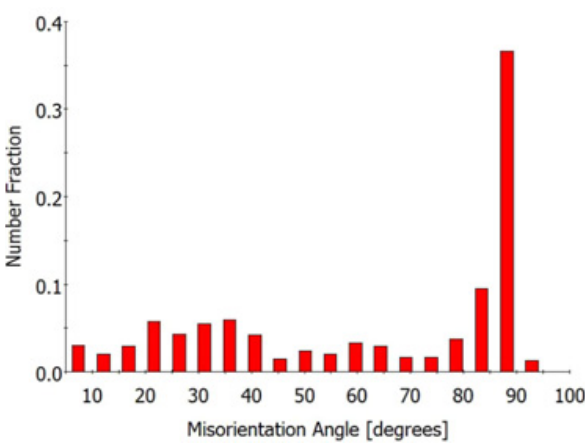

(b)

Figure 3. ) Inverse pole figure in the through thickness direction of the specimen deformed under normal plate impact loading. (b) Calculated misorientation angle distribution for the section in part (a).

should result in the development of some extension twinning. However, the microstructure before impact was devoid of twins (as observed on other samples cut from the same rod).

The inverse pole figure of one region of the deformed sample as measured by EBSD is shown in Fig. 3a. The image shows the through thickness region of the sample such that the impact direction is horizontal in the plane of the figure. Note the grain size of the material, and the presence of many lenticular twins in the deformed microstructure. Note that some grains have multiple twins. Since the microstructure before impact was devoid of twins, the observed twins must have been induced by the impact loading. It is found that most of the twins are extension twins. The morphology of these twins appears to be different than those formed under the high strain rate loading imposed by Kolsky bars and reported earlier by the authors [13]. We now discuss the orientation and size of these impact-induced twins in this pure $\mathrm{Mg}$ material.

Misorientation angle distribution analysis was conducted to characterize the observed twins, and the results are shown in Fig. 3b. The strong peak in the in the $85^{\circ}-90^{\circ}$ range in the misorientation angle distribution chart in Figure 3b corresponds to the extension twin boundaries present in the microstructure.

The average length and width of the twins were measured by analyzing the EBSD and optical images using the commercial image analysis software ImageJ. The

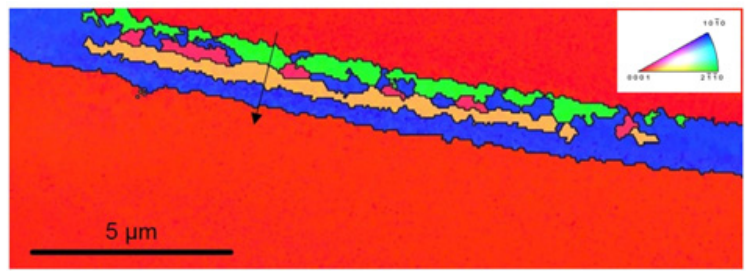

(a)

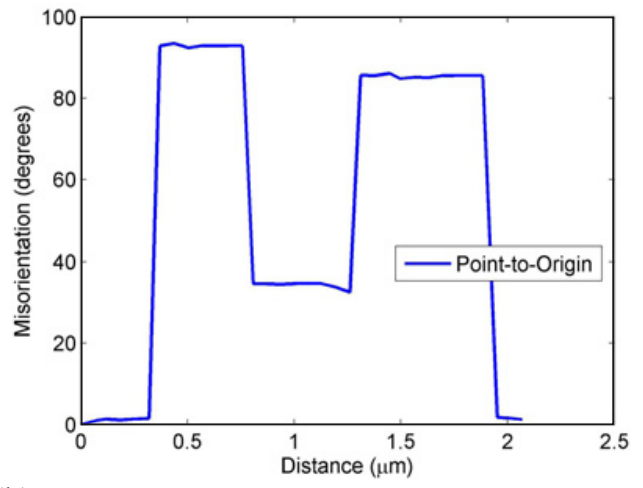

(b)

Figure 4. (a) EBSD inverse pole figure map of a region in a grain within the deformed target. (b) Calculated misorientation angle profile along the black line across the twinned region in the inverse pole figure map shown in (a).

average twin length $\mathrm{L}_{\mathrm{avg}}$ was found to be $27.5 \pm 2.4 \mu \mathrm{m}$ and the average twin width $\mathrm{W}_{\mathrm{avg}}$ was $4.4 \pm 0.7 \mu \mathrm{m}$. From these length measurements and the pulse duration, we can obtain estimates for the twin tip velocity and twin boundary velocity. The maximum amount of time for the twins to grow to the measured sizes is the pulse duration corresponding to the plastic wave propagation. Therefore, the lower bound for the twin tip velocity is given by $\left(\mathrm{v}_{\text {tip }}=\right.$ $\left.L_{\text {avg }} / t_{2}^{\mathrm{pl}}\right)$ and for the twin boundary velocity is given by $\left(\mathrm{v}_{\text {boundary }}=\mathrm{W}_{\text {avg }} / \mathrm{t}_{2}^{\mathrm{pl}}\right)$. In this case, the estimated lower bounds are $\mathrm{v}_{\text {tip }}=20.8 \mathrm{~m} / \mathrm{s}$ and $\mathrm{v}_{\text {boundary }}=3.3 \mathrm{~m} / \mathrm{s}$ for this $\mathrm{Mg}$ alloy under these impact conditions.

To obtain detailed characterizations of the twins developed in plat impact, we performed high resolutions EBSD scans (step size $\leq 0.3 \mu \mathrm{m}$ ) in the regions of interest. The inverse pole figure map of one of the regions studied is presented in Fig. 4a. The different colors represent different crystallographic orientations as represented by the map key in the inset of Fig. 4. It is seen that the twin is in fact composed of different regions that correspond to different orientations. The orientation of the parent grain is represented by the red color and is close to the (0001) orientation. The point-to-origin misorientation profile across the black line in Fig. 4a is illustrated in Fig. $4 b$.

The point-to-origin profile represents the misorientation between each point on the black line with respect to the parent grain orientation. In the line profile, an $\sim 86^{\circ}$ change in the misorientation is observed upon crossing the first extension twin boundary to enter the green twin in Fig. 4a. Upon crossing the boundary between the green and the yellow twins, a smaller misorientation of $\sim 38^{\circ}$ (with respect to the parent orientation) is observed. The yellow 
twin is actually a contraction twin most likely formed through a double twinning mechanism [14]. The blue and green regions are both extension twins that represent the first and second order prismatic planes respectively, and therefore they have a $30^{\circ}$ in-plane misorientation between them.

This morphology of extension twins is significantly different from that observed in the same material under uniaxial stress Kolsky bar loading [13]. Typically only one twin variant is observed to grow significantly and then coalesce with other twins under the Kolsky bar loading. The uniaxial strain condition in the plate impact experiment imposes a constraint on the lateral strains. Therefore, stresses build up in the lateral directions. Within the uniaxial strain configuration an overall higher stress amplitude is necessary to induce significant plastic deformation in this highly plastically anisotropic metal. The uniaxial strain configuration coupled with the extremely short times available for twin nucleation and propagation are likely the major reasons for the observed twin morphology.

\section{Summary}

The dynamic behavior of pure $\mathrm{Mg}$ under normal plate impact loading of microsecond duration was studied. Extension twinning is observed in many grains. The twins are long and thin, and twin coalescence is generally not observed. Estimates of twin tip and twin boundary velocities provide a lower bound on the velocities: $\mathrm{v}_{\text {tip }}=$ $21 \mathrm{~m} / \mathrm{s}$ and $\mathrm{v}_{\text {boundary }}=3.3 \mathrm{~m} / \mathrm{s}$ for this $\mathrm{Mg}$ alloy under these impact conditions.

The observed twins often consist of multiple variants of extension twins. Double twinning is also observed. We speculate that due to the high strain rates (on the order of $10^{4}-10^{5} \mathrm{~s}^{-1}$ ), the high amplitude of stresses due to the uniaxial strain condition and the short times available for deformation $(\sim 1 \mu \mathrm{s})$, nucleation of multiple twin variants is preferred under the plate impact loading instead of the growth and coalescence of the same variant. Therefore, the dynamic loading and nature of the stress state is likely to have a significant effect on the specific deformation mechanisms (especially twinning) activated within magnesium under dynamic loading.

The authors gratefully acknowledge financial support from the NSF DMR Materials World Network program and support from the MEDE CRA, US Army Research Laboratory, to KTR and $\mathrm{KH}$.

This research was sponsored in part by the Army Research Laboratory and was accomplished under Cooperative Agreement Number W911NF-12-2-0022. The views and conclusions contained in this document are those of the authors and should not be interpreted as representing the official policies, either expressed or implied, of the Army Research Laboratory or the U.S. Government. The U.S. Government is authorized to reproduce and distribute reprints for Government purposes notwithstanding any copyright notation herein.

\section{References}

[1] B.L. Mordike and T. Ebert, Mat. Sci. \& Eng. A, 302 (1): 37-45 (2001).

[2] K. Kainer, B.L. Mordike, Wiley-VCH, (2000).

[3] K. Hazeli, A. Sadeghi, M.O. Pekguleryuz, and A. Kontsos, Mat. Sci. \& Eng. A, 589: 275-279 (2014).

[4] G. Wan, B.L. Wu, Y.D. Zhang, G.Y. Sha, and C. Esling, Mat. Sci. \& Eng. A, 527(12): 2915-2924 (2010).

[5] M.T. Tucker, M.F. Horstemeyer, P.M. Gullett, H. El Kadiri, and W.R. Whittington, Scripta Materialia, 60(3): 182-185 (2009).

[6] S. Agnew, W. Whittington, A. Oppedal, H. El Kadiri, M. Shaeffer, K.T. Ramesh, J. Bhattacharyya, R. Delorme, and B. Davis, JOM, 66(2): 277-290 (2014).

[7] K. Eswar Prasad, B. Li, N. Dixit, M. Shaeffer, S.N. Mathaudhu, and K.T. Ramesh, JOM 66(2), pp. 291-304 (2014).

[8] K.T. Ramesh (2008), High rates and impact experiments, Handbook of Experimental Solid Mechanics, Springer US (pp. 929-960).

[9] M.M. Advesian and H. Baker, ASM international, 244 (199).

[10] M.R. Barnett, Mat. Sci. \& Eng. A, Part I, 464, pp. 1-7 (2007).

[11] M.R. Barnett, Mat. Sci. \& Eng.: A, Part II, 464: 8-16 (2007).

[12] L. Jiang, J.J. Jonas, R.K. Mishra, A.A. Luo, A.K. Sachdev, S. Godet, Acta Materialia 55 (11), 3899_ 3910 (2007).

[13] N. Dixit, K.Y. Xie, K.J. Hemker, K.T. Ramesh, Acta Materialia, 87: 56-67 (2015).

[14] M.D. Nave and M.R. Barnett, Scripta Materialia, 51.9: $881-885$ (2004). 